\title{
Virtualização de questões da OBI para o desenvolvimento do pensamento computacional
}

\author{
Ana Flávia S. Aragão Moura ${ }^{1}$, Thiago Henrique C. Tavares ${ }^{1}$, Josilene Aires Moreira ${ }^{1}$, \\ Giorgia de Oliveira Mattos ${ }^{1}$ \\ ${ }^{1}$ Centro de Informática - Universidade Federal da Paraíba (UFPB) \\ João Pessoa - PB - Brasil \\ anaflavia@eng.ci.ufpb.br, thiagoh.coelhotavares@gmail.com, \\ josilene@ci.ufpb.br, giorgia@ci.ufpb.br
}

Resumo. Com intuito de desenvolver o raciocínio lógico, partindo para o processo de desenvolvimento de uma aplicação em 2D para dispositivos móveis, foram desenvolvidos minijogos utilizando conceitos de virtualização aplicados a questões da Olimpíada Brasileira de Informática (OBI). Além de tornar lúdicas e interativas as questões, sendo utilizado também como procedimento de obtenção de informações para o nivelamento dos alunos, a aplicação fomenta o desenvolvimento do pensamento computacional de maneira prática e divertida.

\section{Cenário de uso}

Segundo Quadros (2013, p.15), as olimpíadas científicas são atividades extracurriculares elaborados em diversos países com o intuito de alcançar objetivos comuns, sejam eles cognitivos, afetivos ou sociais. As olimpíadas usam um sistema de competições, dividido por áreas de conhecimento, como por exemplo matemática e informática, e por nível educacional dos alunos. Com o intuito de estimular e promover o estudo dessas áreas do conhecimento aos estudantes, contribuindo para o incentivo de seu ingresso nessas áreas. Na modalidade iniciação da Olimpíada Brasileira de Informática as provas são realizadas com papel e lápis, com questões sobre lógica no formato de múltipla escolha.

De acordo com Prensky (2001, p. 2), "os estudantes de hoje não são mais as mesmas pessoas para as quais nosso sistema educacional foi desenvolvido". Diante disso, propomos uma solução de aplicação móvel, com intuito de tornar mais dinâmico e instigante a resolução de questões de lógica, como objeto de aprendizagem para o ensino do exercício do Pensamento Computacional para alunos do Ensino Fundamental e Médio. A principal estratégia desta ferramenta é a abordagem em forma de jogos interativos. Destaca Bristot et al. (2017, p. 9), "a falta de representatividade feminina nos games se deve a indústria identificar o público masculino como o maior consumidor de games. Essa falta de representatividade e diversidade acaba por excluir as mulheres como consumidoras de games bem como gerar preconceitos e estereótipos negativos". Sabendo disso, a aplicação tem como requisito essencial ser atrativa tanto ao público feminino como ao masculino, objetivando diminuir as desigualdades de atuação na área da Ciência da Computação. 
Segundo Borin (1996) quando, na prática pedagógica é proposta uma aprendizagem partindo do lúdico, os resultados são mais positivos. Com os jogos, na participação ativa e direta do aluno é estimulado o raciocínio lógico e o pensamento independente, a criatividade e capacidade de resolver problemas. Assim, os recursos lúdicos auxiliam no ensino de disciplinas que utilizam de raciocínio lógico como matemática, impactando diretamente o aprendizado e produtividade dos alunos.

\section{Desenvolvimento}

Segundo Santos et al (2014), “O processo de virtualização é compreendido, como o processo pelo qual, os jogos tradicionais, são recriados em versões digitais, de maneira que não percam seus aspectos pedagógicos e psicopedagógicos, que os levaram a ser utilizados de forma positiva como ferramenta auxílio ao processo de ensino e aprendizado". O objeto de virtualização foram as questões de múltipla escolha da OBI, pensando na interação dinâmica entre aluno e o jogo. Foram escolhidas oito questões no total, compreendendo diferentes graus de dificuldade mas que ao mesmo tempo envolvessem situações e objetos familiares à realidade de alunos do ensino fundamental e médio.

As questões abordam diferentes aspectos da lógica e da matemática, tendo o principal enfoque no desenvolvimento de habilidades do pensamento computacional. Para Brackmann (2017), "O Pensamento Computacional é uma distinta capacidade criativa, crítica e estratégica humana de saber utilizar os fundamentos da Computação nas mais diversas áreas do conhecimento, com a finalidade de identificar e resolver problemas colaborativamente através de passos claros de tal forma que uma pessoa ou uma máquina possam executá-los eficazmente". Dentro das principais habilidades exploradas estão a organização lógica e análise de dados, divisão do problema em fragmentos menores, pensamento iterativo, simbólico e abstrato, bem como a reformulação de problemas em uma série de etapas ordenadas.

No desenvolvimento da aplicação foi utilizada a ferramenta Unity, também conhecido como Unity 3d. É um motor de jogos e ambiente integrado de desenvolvimento multiplataforma criado pela Unity Technologies. Foi utilizada a versão gratuita da Unity e a aplicação foi desenvolvida utilizando recursos em $2 \mathrm{D}$, pois a aplicação tem apenas duas dimensões. A Unity é uma interface de desenvolvimento dinâmica que dá suporte a uma vasta quantidade de modelos $2 \mathrm{D}$ e $3 \mathrm{D}$ de outras aplicações

Além do fácil manuseio e curta curva de aprendizado quando comparada a outras ferramentas como Unreal Engine por exemplo, a Unity ainda oferece suporte a portabilidade de aplicações para diversos meios digitais. Isso significa que com pequenos a nenhum ajuste, uma mesma aplicação programada em uma só linguagem, no caso escolhemos C\# pela familiaridade com a linguagem, pode ser executada em uma 
VIII Congresso Brasileiro de Informática na Educação (CBIE 2019)

Anais dos Workshops do VIII Congresso Brasileiro de Informática na Educação (WCBIE 2019)

vasta quantidade dispositivos, como navegadores de computadores, dispositivos android, IOS ou até mesmo em sistemas operacionais de algumas Smart TV's.

Uma das primeiras etapas, de vital importância para o sucesso no desenvolvimento, é a identificação de requisitos do software. Segundo Swebok (2004, p. 33-36) é de uma prioridade que deve ser exibida ou adaptada na condição de resolver algum problema em particular no mundo real. Com o intuito de fazer uma descrição completa do que o sistema deve fazer, mas também atingir essas metas no tempo determinado. Porém, quando tratamos do desenvolvimento de uma aplicação denominada jogo, também devemos ter atenção a outras etapas desse processo como o design de jogos.

Segundo Schell (2008) "O design de jogos é o ato de decidir o que um jogo deve ser.”. Neste contexto, A Tétrade Elementar (Fig. 1) é um conceito interessante que pode ser encontrado no livro The Art of Game Design, por Schell (2008), que descreve os elementos básicos e necessários para a estrutura de um jogo. Os elementos estão divididos em quatro categorias: Mecânica, Narrativa, Estética e Tecnologia. Na aplicação descrita exploramos as categorias mecânica, estética e tecnologia, não nos preocupando tanto com a narrativa, que deverá ser trabalhada no futuro..

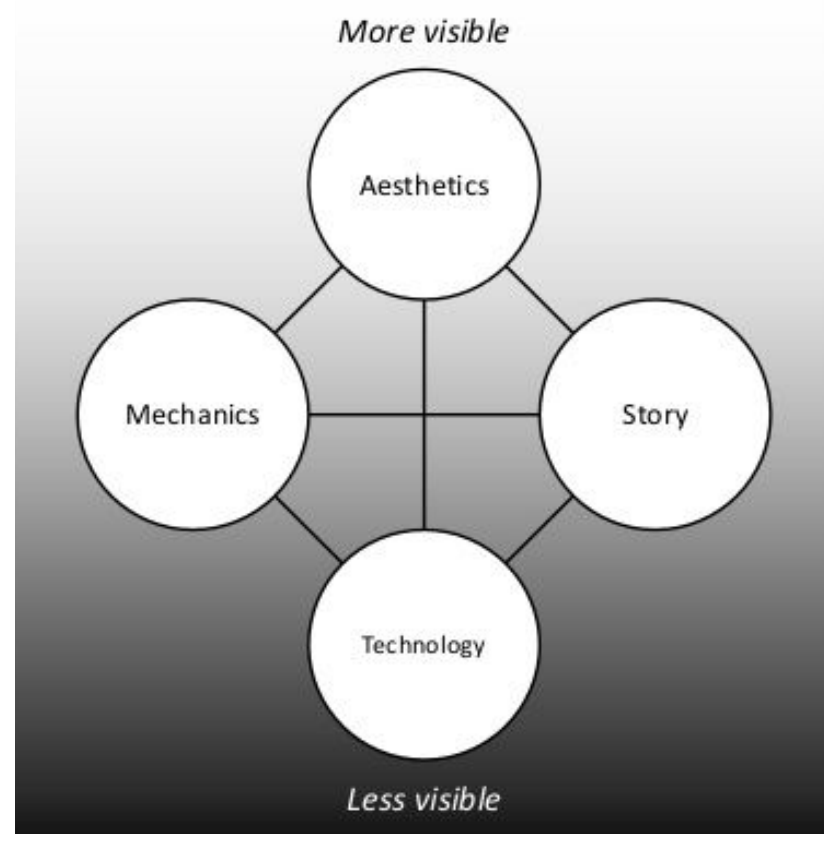

Figura 1. A Tétrade Elementar. Fonte: The Art of Game Design, Schell (2008).

\section{Apresentação do Software}

O aplicativo apresentado neste trabalho, disponível para a plataforma Android em $<$ link download>, é constituído de perguntas da OBI que foram virtualizadas em função de 
VIII Congresso Brasileiro de Informática na Educação (CBIE 2019)

Anais dos Workshops do VIII Congresso Brasileiro de Informática na Educação (WCBIE 2019)

torná-las mais lúdicas e interessantes para o público-alvo, alunos do ensino fundamental e médio. Nas telas, é proposto o uso de diferentes mecânicas de interação para estimular o raciocínio lógico e engajar o aluno no jogo.

De forma geral, quando falamos em jogos, são chamadas de mecânicas os conjuntos de regras ou métodos criados para proporcionar interação entre o usuário e o jogo. Criar um projeto em formato de coletânea de minijogos introduz uma grande facilidade em diversificar as maneiras de interagir com o produto, tornando assim a experiência ainda mais única e interessante na perspectiva do usuário.

$\mathrm{Na}$ fase inicial do desenvolvimento do projeto, foram selecionados dois tipos de mecânicas principais. Devido à natureza mimética da virtualização de experiências, a escolha das mecânicas não deve causar interferência no conteúdo focal das questões, em seus padrões de dificuldade, nem muito menos na forma de resolvê-las. Para tal, foram selecionadas as mecânicas de "clique e arraste" e questões de múltipla escolha.

A mecânica de "clique e arraste", demonstrada nas Figuras 2 e 3, funciona de maneira a arrastar blocos até posições específicas com o intuito de gerar sequências de objetos ou fatos de acordo com a lógica da questão. Sendo assim, a pontuação é dada de acordo com o tempo gasto para resolução do desafio. A pontuação máxima é 100 e ao decorrer do tempo diminui, podendo chegar até 50. Caso o jogador tenha construído a alternativa incorreta, em outras palavras arrastar as os objetos na sequência incorreta, não serão atribuídos pontos e será mostrada a próxima tela.

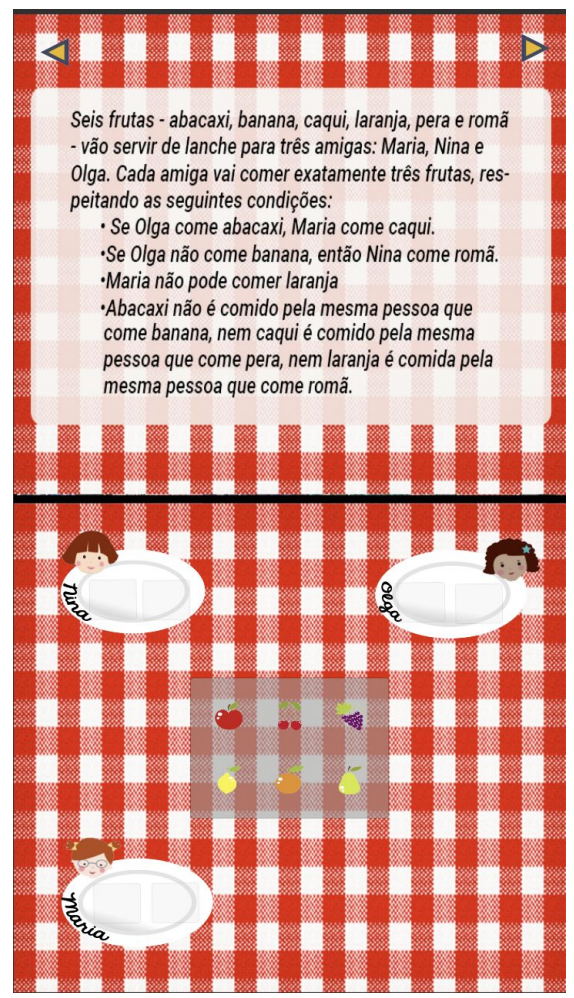

Figura 2. Fase das frutas. Fonte: Aplicativo proposto pelos autores, 2019. 
VIII Congresso Brasileiro de Informática na Educação (CBIE 2019)

Anais dos Workshops do VIII Congresso Brasileiro de Informática na Educação (WCBIE 2019)

Tanto na fase das frutas, exibida na Figura 2, quanto na fase das cidades, na Figura 3, usamos a mecânica de mover objetos a espaços vazios, de acordo com a lógica da questão. No primeiro caso, as frutas são movidas até os pratos, de modo a atender as restrições descritas no problema. No segundo caso, as cidades são movidas de modo a criar uma lista lógica, de acordo com a ordem das cidades que serão visitadas.

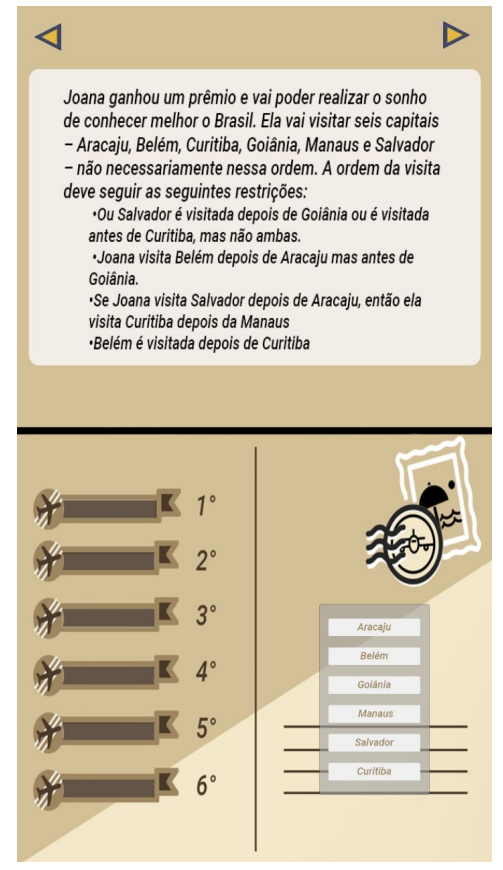

Figura 3. Fases das cidades. Fonte: Aplicativo proposto pelos autores, 2019.

Outra mecânica utilizada na aplicação é o uso de questões de múltipla escolha, representada nas Figuras 4 e 5. Nesse tipo de questão, o usuário resolve operações lógicas e matemáticas mentalmente e seleciona a opção correta. Com o decorrer do tempo, a pontuação é reduzida de 100 pontos até chegar a 50, como nas fases apresentadas anteriormente. 
VIII Congresso Brasileiro de Informática na Educação (CBIE 2019)

Anais dos Workshops do VIII Congresso Brasileiro de Informática na Educação (WCBIE 2019)

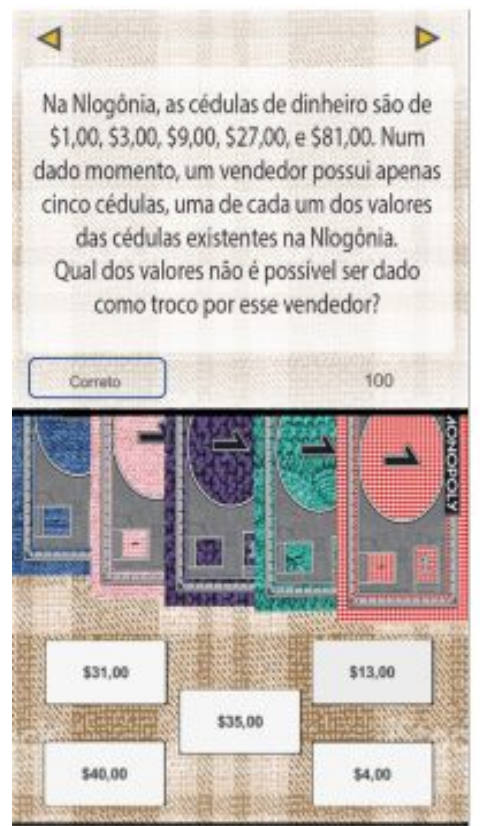

Figura 4. Correto e Pontuação.

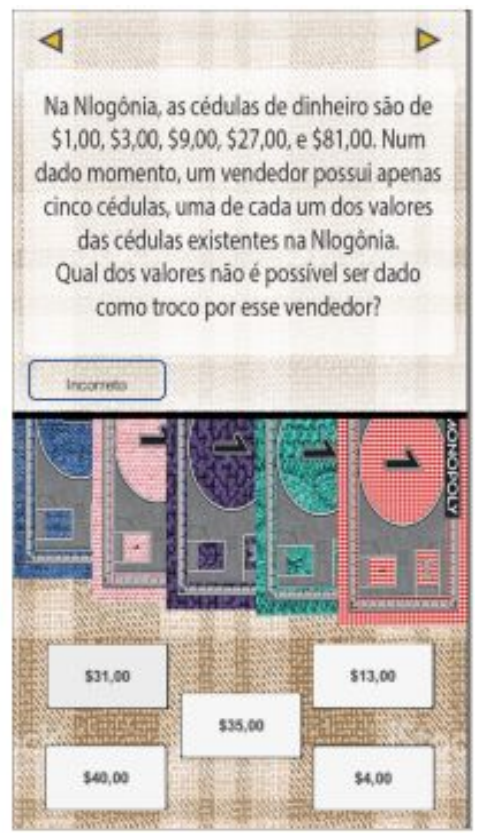

Figura 5. Incorreto.

Fonte: Aplicativo proposto pelos autores, 2019.

Outro ponto importante em mídias em formato de jogos é o elemento de feedback, ou seja, retroalimentação de informação ao usuário. Nas Figuras 4 e 5 são demonstrados os comportamentos de acerto e erro durante a execução do jogo. Quando o usuário seleciona a resposta incorreta, é exibida a mensagem de "Incorreto", mas sem a pontuação para que o jogador continue tentando e não se sinta desmotivado, até obter uma pontuação melhor. Ao selecionar a opção correta, é exibida a mensagem de confirmação e sua pontuação.

\section{Considerações finais}

As atividades do desenvolvimento da aplicação para dispositivos portáteis que objetiva além de fazer uma medição inicial, aprimorar as habilidades de pensamento computacional de alunos e alunas do ensino médio e fundamental da Paraíba. A pesquisa teve como principal propósito desenvolver uma aplicação que em seu estado de funcionamento, pudesse ser aplicada diretamente nas escolas ou nas salas de aula cedidas ao projeto "Meninas na computação" dentro da própria Universidade Federal da Paraíba.

As questões já virtualizadas e devidamente programadas encontram-se atualmente em fase de testes para que seja iniciada a aplicação nas escolas parceiras do projeto. Faz-se necessária tal etapa para que a experiência do usuário não seja afetada por bugs, erros ou falhas do sistema, que também prejudicam a obtenção de resultados do experimento. 
VIII Congresso Brasileiro de Informática na Educação (CBIE 2019)

Anais dos Workshops do VIII Congresso Brasileiro de Informática na Educação (WCBIE 2019)

Dos resultados já obtidos com outras aplicações exploradas no projeto em conjunto com as questões que previamente eram aplicadas em formato de teste, é possível notar a diferença no comportamento dos alunos quando expostas à ferramentas digitais como "Scratch" e "LightBot". O nível de aceitação tem sido muito positivo no caso de ferramentas mais lúdicas com a sensação de atividade prática, fazendo com que o aprendizado se torne mais fácil e interessante na visão dos alunos.

Pretendemos expandir para outras escolas do estados, para que o aplicativo seja utilizado de forma gratuita e ativa, envolvendo diretamente alunos e professores para o auxílio na aprendizagem e incentivo, da lógica e do pensamento computacional. Contribuindo para a sociedade de forma positiva, na educação.

\section{Referências}

Brackmann, C. (2017) "Desenvolvimento do Pensamento Computacional Através de Atividade Desplugadas na Educação Básica”, Universidade Federal do Rio Grande do Sul (UFRGS), Porto Alegre, RS, Brasil, http://hdl.handle.net/10183/172208, Julho.

Quadros, A., Fátima, A., Martins, D., Silva, F., Freitas-Silva, G., Aleme, H., Oliveira, S., Andrade, F., e Santos, L. (2013) "Ambientes colaborativos e competitivos: o caso das olimpíadas científicas. Revista de Educação Pública", http://periodicoscientificos.ufmt.br/ojs/, Julho.

Prensky, M. (2001) Digital Natives, Digital Immigrants. On the Horizon, MCB University Press, v. 9, n. 5.

Borin, J. (1996) Jogos e Resolução de problemas: uma estratégia para as aulas de matemática. São Paulo: IME-USP.

IEEE. (2004) Guide to the Software Engineering Body of Knowledge (SWEBOK). Version. http://www.swebok.org, Julho.

Schell, J. (2008), The Art of Game Design, Morgan kaufmann publishers.

Santos, W. O., Silval, A. P., Junior, S. G. C. (2014) Conquistando com o Reto1: Virtualização de um Jogo para o Ensino de Matemática XXV, Simpósio Brasileiro de Informática na Educação.

Bristot, P. C., Pozzebon, E., Frigo, L. B. (2017) "A representatividade das mulheres nos Games. SBC - Proceedings of SBGames, https://www.sbgames.org/ sbgames2017/papers/CulturaFull/175394.pdf, Agosto. 
VIII Congresso Brasileiro de Informática na Educação (CBIE 2019)

Anais dos Workshops do VIII Congresso Brasileiro de Informática na Educação (WCBIE 2019) 